\title{
Diagnósticos diferenciais das deficiências cognitivas em idosos
}

\author{
Differential diagnoses of cognitive impairments in the elderly \\ Diagnósticos diferenciales de deterioro cognitivo en ancianos
}

Bruna de Sousa Silva ${ }^{1 *}$, Isabella dos Santos Zanetti ${ }^{1}$, Patrícia Peres de Barros ${ }^{1}$, Letícia Ton de Souza ${ }^{1}$, Leonardo Brandão Barreto ${ }^{1}$.

\section{RESUMO}

Objetivo: Realizar uma revisão bibliográfica a fim de identificar as possíveis etiologias das deficiências cognitivas em idosos e seus diagnósticos diferenciais. Revisão bibliográfica: A demência é caracterizada por comprometimento gradual da função cognitiva que interfere nas atividades sociais e profissionais, uma vez que, os indivíduos apresentam uma maior propensão ao desenvolvimento de incapacidades funcionais, podendo ser causa importante de redução na qualidade de vida dos idosos e de seus familiares. $O$ estudo revela a existência de uma grande variedade de etiologias das deficiências cognitivas em idosos, sendo classificadas em demências degenerativas e demências não degenerativas. As demências degenerativas (ou primárias) incluem a doença de Alzheimer, a demência por corpos de Lewy e a demência frontotemporal, dentre outras. As demências não degenerativas (ou secundárias) abrangem inúmeros subtipos dentre os quais a demência vascular, deficiência de vitaminas, depressão e insuficiência renal. Considerações finais: As deficiências cognitivas representam um grande problema de saúde pública, com consequências significativas para os idosos e seus familiares. Diante da grande variedade de etiologias das deficiências cognitivas é fundamental o conhecimento de seus diagnósticos diferenciais.

Palavras-chave: Disfunção cognitiva, Idoso, Doença de Alzheimer.

\begin{abstract}
Objective: Conduct a bibliographic review in order to identify the possible etiologies of cognitive impairments in the elderly and their differential diagnoses. Bibliographic review: Dementia is characterized by a gradual impairment of cognitive function that interferes with social and professional activities, since individuals present a greater propensity to the development of functional disabilities, and, in addition, it can cause a reduction in the quality of life of the elderly and their families. The study reveals the existence of a wide variety of etiologies of cognitive impairments in the elderly, being classified into degenerative dementia and non-degenerative dementia. Degenerative (or primary) dementias include Alzheimer's Disease, Lewy Body Dementia and Frontotemporal Dementia, among others. Non-degenerative (or secondary) dementias comprise numerous subtypes, including Vascular Dementia, vitamin deficiency, depression and kidney failure. Final considerations: Cognitive disabilities represent a major public health problem, with significant consequences for the elderly and their families. In view of the wide variety of etiologies of cognitive impairments, knowledge of their differential diagnoses is essential.
\end{abstract}

Key words: Cognitive dysfunction, Elderly, Alzheimer's disease.

\section{RESUMEN}

Objetivo: Realizar una revisión de la literatura con el fin de identificar las posibles etiologías de los deterioros cognitivos en el anciano y sus diagnósticos diferenciales. Revisión bibliográfica: La demencia se caracteriza por un deterioro gradual de la función cognitiva que interfiere con las actividades sociales y profesionales, ya que los individuos presentan una mayor propensión al desarrollo de discapacidades funcionales, y, además, puede provocar una reducción en la calidad de vida de las personas mayores y sus familias. El estudio revela la existencia de una amplia variedad de etiologías de deterioro cognitivo en el anciano, clasificándose en demencia degenerativa y demencia no degenerativa. Las demencias degenerativas (o primarias) incluyen la

${ }^{1}$ Faculdade Dinâmica do Vale do Piranga (FADIP), Ponte Nova - MG. *E-mail: brunadesousass@gmail.com 
enfermedad de Alzheimer, la demencia con cuerpos de Lewy y la demencia frontotemporal, entre otras. Las demencias no degenerativas (o secundarias) abarcan numerosos subtipos que incluyen demencia vascular, deficiencia de vitaminas, depresión e insuficiencia renal. Consideraciones finales: Las discapacidades cognitivas representan un importante problema de salud pública, con importantes consecuencias para las personas mayores y sus familias. Dada la amplia variedad de etiologías de las alteraciones cognitivas, el conocimiento de sus diagnósticos diferenciales es fundamental.

Palabras clave: Disfunción cognitiva, Anciano, Enfermedad de Alzheimer.

\section{INTRODUÇÃO}

O déficit cognitivo ou demência é caracterizado por declínio cognitivo persistente, que interfere nas atividades sociais ou profissionais do indivíduo, independente de alterações do nível de consciência (BEZERRA PK, et al., 2016). Os tipos mais comuns de demência são a Doença de Alzheimer (DA), demência vascular, demência frontotemporal e demência dos corpos de Lewy (MENEZES AV, et al., 2016). Outra condição que deve ser considerada é o comprometimento cognitivo leve que apresenta como característica principal prejuízo da memória episódica sem outros déficits cognitivos e funcionais, o que representa risco elevado de desenvolvimento da DA (MENEZES AV, et al., 2016).

As síndromes demenciais podem ser classificadas em degenerativas e não degenerativas. As não degenerativas são decorrentes de deficiências nutricionais, acidentes vasculares, processos infecciosos, traumatismos, dentre outras condições. Já entre as degenerativas a principal a ser considerada é a DA (BEZERRA PK, et al., 2016).

Entre as principais doenças não transmissíveis da população idosa, destacam-se os quadros demenciais. A demência, apesar de ter casos manifestos em pacientes considerados de baixa idade, acomete em sua maioria os idosos, sobretudo aqueles acima de 65 anos (SANTOS MD e BORGES SM, 2015). Durante o processo de envelhecimento, aproximadamente $15 \%$ das pessoas desenvolvem déficit cognitivo progressivo, em média $5 \%$ das pessoas acima de 65 anos e $20 \%$ acima de 80 anos desenvolvem demência moderada a grave (BERTOLDI JT, et al., 2015).

Várias capacidades cognitivas sofrem alterações com a idade, como o declínio cognitivo de processos fisiológicos normais ou um estágio de transição para a demência (BRANDÃO BMLS, et al., 2020). O aumento da prevalência dessas condições pode ser explicado pelo envelhecimento da população, em que se observa um aumento da prevalência de doenças crônicas não transmissíveis e maior procura por atendimento médico (BRANDÃO BMLS, et al., 2020).

Estima-se que a população com 60 anos ou mais, equivalente a $10 \%$ da população total em 2010, atinja $13,7 \%$ da população em 2020 e $23,8 \%$ em 2040, o que colabora para a mudança da estrutura etária populacional (SANTOS CS, et al., 2020). O envelhecimento faz com que os indivíduos apresentem declínio cognitivo, sendo importante o diagnóstico oportuno da demência para promover o enfrentamento adequado, tratar ou retardar a progressão dos sintomas cognitivos e neuropsiquiátricos (PRADO M, et al., 2018).

Essas condições trazem como consequências uma maior propensão ao desenvolvimento de incapacidades funcionais, como a dificuldade em tomar decisões, desempenhar tarefas e associar informações (NUNES WA, et al., 2016; FAGUNDES TA, et al., 2017). O comprometimento cognitivo pode ainda causar a redução da qualidade de vida do idoso e de seus familiares, o aumento da procura por atendimento médico e a redução do humor associado a depressão (NUNES WA, et al., 2016; FAGUNDES TA, et al., 2017).

A incapacidade cognitiva é um tema que requer mais estudos, uma vez que são inúmeros os fatores que a predispõem. Além disso, existe a necessidade de maior atenção às variáveis predisponentes, para que seja possível traçar metas de atenção à saúde dessa população (PRADO M, et al., 2018). Tendo em vista essas questões, esse estudo tem como objetivo realizar uma revisão bibliográfica a fim de identificar as possíveis etiologias das deficiências cognitivas em idosos e seus diagnósticos diferenciais. 


\section{REVISÃO BIBLIOGRÁFICA}

As síndromes demenciais podem ser classificadas em duas categorias: degenerativas e não degenerativas. As demências degenerativas (ou primárias) são caracterizadas por acometimento progressivo da cognição, o qual inclui a DA, a demência por corpos de Lewy e a demência frontotemporal, dentre outras. As demências não degenerativas (ou secundárias) abrangem inúmeros subtipos dentre os quais a demência vascular, deficiência de vitaminas, depressão e insuficiência renal (ARAÚJO C, 2010; FORNARI LHT, et al., 2010). Na prática clínica, as quatro causas mais frequentes de demência são a DA, demência vascular, demência por corpos de Lewy e a demência frontotemporal (CARAMELLI P E BARBOSA MT, 2002).

\section{Demências degenerativas}

\section{Doença de Alzheimer}

A DA é caracterizada como uma síndrome cerebral degenerativa que apresenta destruição dos neurônios gerando perda progressiva e incapacitante, sendo a causa mais comum de demência em idosos. A DA afeta o déficit cognitivo e funcional, manifestando na fase leve com falha na memória recente, desorientação temporal e espacial e perda de interesse em atividades cotidianas. $\mathrm{Na}$ fase moderada, é observada a incapacidade de lembrar nome de pessoas próximas, dificuldade em realizar compras, cozinhar, cuidar da casa, alterações comportamentais e de linguagem. Já na fase grave, há incontinência urinária/fecal e prejuízo da marcha (SANTOS MD E BORGES SM, 2015).

A DA possui etiologia indeterminada, mas podem ser verificadas alterações neurobioquímicas e neuropatológicas, como atrofia cortical difusa, degeneração neurovascular, perdas neuronais e sinápticas, estresse oxidativo, neuroinflamação, desregulação de cálcio, deficiência de mitocôndrias e alterações da hemostase metálica. Podem ainda ser observadas a presença de placas senis agrupada da proteína beta amiloide e massas neurofibrilares intracelulares nas amídalas cerebelosas, hipocampo e no córtex entorrinal do lóbulo temporal (FALCO AD, et al., 2016).

O diagnóstico da DA é feito pela exclusão de outras patologias, através de anamnese detalhada incluindo o histórico familiar e exames laboratoriais como hemograma completo, creatinina, sódio, potássio, cálcio, dosagem de vitamina B12, sorologia para sífilis e função hepática. Na tomografia computadorizada ou ressonância magnética são observados sulcos corticais largos e ventrículos cerebrais maiores. No exame microscópico é possível encontrar falha neuronal, alteração sináptica intensa, presença de placas senis e neurofibrilares (CAETANO LAO, et al., 2017).

A DA não tem cura e sua terapêutica objetiva retardar a evolução e melhorar o déficit cognitivo. Atualmente, existem quatro fármacos disponíveis, sendo eles: galantamina, rivastigmina, memantina e donepezil. A galantamina atua como agonista de receptores nicotínicos, gerando maior liberação de acetilcolina na fenda sináptica. A rivastigmina é indicada para o tratamento da DA leve a moderada que atua inibindo as colinesterases. A memantina age nos receptores de glutamato evitando um influxo de cálcio, e por fim, a donepezil aumenta a disponibilidade de acetilcolina na fenda sináptica (DIAS KST, et al., 2015).

\section{Demência por Corpos de Lewy}

A Demência de Corpos de Lewy (DCL) pode ser caracterizada por uma síndrome flutuante, com sinais parkinsonianos, síncope, distúrbio comportamental de Movimento Rápido dos Olhos (REM), alucinações visuais e hipersensibilidade a antipsicóticos. Outros sintomas também foram descritos, como depressão psicótica, catatonia e transtornos delirantes. Possui déficits colinérgico e dopaminérgico, e cerca de $50 \%$ dos pacientes costumam apresentar sensibilidade neuroléptica grave, com piora da cognição, sedação, parkinsonismo de início agudo, além de sintomas que lembram a síndrome neuroléptica maligna. $O$ tratamento deve ser individualizado e cauteloso, podendo-se optar pelos inibidores da acetilcolinesterase para tratamento dos sintomas cognitivos, comportamentais e psicóticos (MENDONÇA FJP, et al., 2020).

\section{Demência Frontotemporal}

A Demência Frontotemporal (FTD) é uma doença neurodegenerativa caracterizada pelo envolvimento dos lobos frontal e temporal, associadas à perda neuronal e gliose. É considerada a demência mais comum em 
adultos com menos de 65 anos, geralmente se apresentando entre 50-60 anos. Estima-se que sua prevalência seja de 15-22 casos/100.000 habitantes. A FTD pode ser subdividida em três síndromes: uma caracterizada por alterações comportamentais e controle executivo e duas com alterações da linguagem. As variantes que tem um comprometimento diferencial da área da linguagem em relação ao resto das funções cognitivas, podem ser agrupadas sob o termo "afasia progressiva primária" (APP), no entanto, podem estar sobrepostas em outras síndromes motoras, como no parkinsonismo atípico e na esclerose lateral amiotrófica (LANCHO MCP e BERCIANOS SG, 2020).

A FTD é caracterizada por alterações comportamentais (desinibição, hiperoralidade, movimentos estereotipados e compulsões), alterações de personalidade e afetivas (apatia, inércia, perda de empatia), disfunção executiva e da linguagem, atrofia cerebral frontal e/ou temporal anterior. A FTD tem influência familiar, visto que $40 \%$ dos pacientes têm um parente com essa condição. (RAMOS C, et al., 2020).

O diagnóstico se baseia na avaliação cognitiva, podendo ser utilizados instrumentos de triagem. O Exame do Estado Minimental (MEEM), apesar de ser o mais difundido, não é sensível para a detecção nesses casos, ao contrário do Exame Cognitivo de Addenbrooke (ACE), que tem boa sensibilidade e o distingue da DA. Algumas baterias de avaliação neuropsicológica, podem verificar dificuldades executivas e outras cognitivas, estabelecendo diagnósticos diferenciais. É importante também colher as informações de familiares e cuidadores acerca da presença de sintomas neuropsiquiátricos (SEPULVEDA-IBARRA C, 2020).

A ressonância magnética ajuda a excluir outras causas de declínio cognitivo, podendo evidenciar atrofia, que geralmente afeta a parte anterior do lobo frontal e temporal, respeitando a margem posterior do giro temporal superior, o córtex peri-rolândico, os lobos parietal e occipital. A neuroimagem também pode evidenciar as circunvoluções da "lâmina de faca", gliose na substância branca subcortical subjacente, e assimetria direita-esquerda devido ao maior envolvimento em um hemisfério. O tratamento da FTD se baseia em sintomáticos e de suporte, tendo prognóstico de seis a onze anos desde o início da clínica (PANTOJA MAG, et al., 2020).

\section{Demências não degenerativas}

\section{Demência vascular}

A demência vascular é a segunda causa mais frequente dentre as doenças cerebrais orgânicas, sendo os principais fatores de risco a aterosclerose, idade, diabetes Mellitus, hipertensão arterial sistêmica, hipercolesterolemia, hipertrigliceridemia e os demais fatores de risco neurovasculares (SANTOS MAO, et al., 2018).

A doença dos pequenos vasos é decorrente da lipo-hialinose, arteriolosclerose e da necrose fibrinoide. A aterosclerose das artérias perfurantes ou a hipertensão arterial, podem causar a oclusão ou estreitamento arterial, com isquemia subsequente. Está relacionada ao aumento na permeabilidade da barreira hematoencefálica, resultando na infiltração de componentes do plasma e células inflamatórias, afetando as paredes vasculares e tecidos subjacentes, levando a perda da autorregulação vascular através da lesão do musculo liso, redução luminal e oclusão. A ressonância magnética pode evidenciar pequenos infartos subcorticais, que geralmente ocorrem nas artérias perfurantes. Está relacionada ao declínio clínico e cognitivo do envelhecimento (SHIBUYA M, et al., 2017).

O Acidente Vascular Cerebral (AVC) e a demência compartilham os mesmos fatores predisponentes. Além disso, representam risco uma para a outra, visto que a neurodegeneração compromete a microcirculação, do mesmo modo, as alterações na microcirculação aceleram a neurodegeneração. A disfunção endotelial pode levar a desnutrição dos neurônios e de outras células cerebrais, prejudicando a eliminação de produtos tóxicos do cérebro, como a beta amiloide. Da mesma forma, alguns produtos secretados por neurônios podem paralisar os capilares (HACHINSKI V, 2018).

O tratamento da demência vascular pode ser dividido em prevenção primária (controle dos fatores de risco), prevenção secundária (prevenção da exacerbação ou extensão das lesões) e prevenção terciária (tratamento sintomático de problemas cognitivos e comportamentais) (CUSTODIAN N, 2016). Os fatores de risco podem interagir entre si e com fatores genéticos, contribuindo para o desenvolvimento da demência. 
Sugere-se que alguns alimentos possam interferir na capacidade de regeneração celular, atuando nas vias patogênicas da demência, como as vitaminas do complexo $B$, folato, vitamina $D$, os antioxidantes, o ômega3 , cafeína e outros. Como forma de reduzir o risco de demência, é importante o controle dos fatores de risco vascular, como a modificação no estilo de vida, alimentação saudável e o tratamento de comorbidades associadas (CARDOSO SA e PAIVA I, 2017).

\section{Deficiência de Vitamina B12}

A vitamina B12, também denominada cianocobalamina, é um micronutriente essencial cuja maior fonte são as proteínas animais. Sua deficiência pode ocasionar alterações no Sistema Nervoso Central (SNC), como neuropatia periférica, depressão, degeneração cognitiva e, consequentemente, demências (BRAUN NM, et al., 2017).

Essa relação com o SNC pode ser explicada pelo papel da vitamina B12 como cofator essencial para duas enzimas que estão envolvidas no metabolismo da homocisteína (Hcy): a L-metilmalonilCoa mutase e a metionina sintase. A metionina sintase converte a Hcy em metionina, formando ao final a S-adenosilmetionina (SAM), que é essencial para reações de manutenção da mielina. Dessa forma, indivíduos com deficiência de vitamina B12, poderão apresentar redução da SAM e aumento da Hcy, com consequentes efeitos desmielinizantes no SNC. A L-metilmalonilCoa mutase tem papel importante na eliminação da Hcy e consequente metionina, cujo excesso é crucial nos danos neurológicos causados pela deficiência de vitamina B12 (BRAUN NM, et al., 2017; BECK WO, 2018).

A deficiência de vitamina B12 é comum em idosos e tende a aumentar com a idade. Entre as suas causas podemos citar a redução do fator intrínseco e da capacidade absortiva, ingesta inadequada, interação com múltiplos fármacos e o uso de álcool (MARTINS JT, et al., 2017; FRANÇA VF, et al., 2016). Além das alterações no SNC, essa deficiência pode ocasionar alterações hematopoéticas, alterações da mucosa e do trato digestivo. A literatura considera os valores inferiores a $200 \mathrm{pg} / \mathrm{ml}$ como baixos, sendo necessário o tratamento com administração deste nutriente (BRAUN NM, et al., 2017). Diante da sua gravidade e associação com outras doenças, é fundamental o diagnóstico precoce da deficiência de vitamina B12, com a finalidade de prevenir maiores danos e extensão da doença (BRAUN NM, et al., 2017).

\section{Hipovitaminose D}

A vitamina $D$ é sintetizada pela pele por meio da exposição à luz solar e uma pequena porção vem de fontes dietéticas. Poucos alimentos apresentam quantidades significativas dessa vitamina, como o salmão, atum, sardinha, cogumelos e ovo. A vitamina D pode ser encontrada nas formas de ergocalciferol (vitamina D2) e de colecalciferol (vitamina D3). Elas são semelhantes, pois são sintetizadas na pele através de seus precursores, utilizando a radiação solar. Após serem ingeridas, seguem a mesma via de metabolismo no fígado para dar origem a 25-hidroxivitamina-D. Alguns fatores de risco para hipovitaminose $D$ estão relacionados a pouca exposição solar, pele negra, uso de protetor solar, má absorção alimentar, idade avançada e outros. A vitamina $D$ atua na regulação fisiológica osteomineral, especialmente do fósforo e cálcio, além de participar da modulação imunológica, síntese de interleucinas inflamatórias, multiplicação e diferenciação celular e outros processos (JORGE AJL, et al., 2018).

Estudos sugerem a capacidade dos alimentos na modificação do risco, atraso da ocorrência e progressão da demência, estando os baixos níveis de vitamina $D$ associados a doenças cerebrovasculares e a maior risco de demências (CARDOSO SA e PAIVA I, 2017). A vitamina D é importante no desenvolvimento do cérebro e maturação dos receptores de vitamina $D$ (VDRs) que estão presentes em várias áreas do cérebro, incluindo as relacionadas à aprendizagem e memória (SANTOS CS, et al., 2020). Essa vitamina possui ação neuroprotetora, como efeitos antioxidantes e antiinflamatórios, evitando a excitotoxicidade do cálcio, eliminação de $A \beta$ e a indução da expressão gênica de neurotransmissores. (OLIVEIRA ACR, et al., 2018).

Sendo a vitamina $D$ um fator modificável, deve-se abrir as perspectivas para políticas de saúde pública, visto que é passível de intervenção, especialmente a comportamentos saudáveis. Além disso, deve ser investigada a associação entre baixos níveis de vitamina $\mathrm{D}$ em pacientes com o declínio cognitivo (SANTOS CS, et al., 2020). 


\section{Depressão}

A depressão é uma condição multifatorial, envolvendo aspectos psicológicos, biológicos e socioculturais que pode acometer o funcionamento cognitivo, afetando a qualidade de vida. Nos idosos essa patologia vem crescendo, visto que eles são mais propensos a estarem em situações de perdas, como declínio físico, de saúde e abstenção de status ocupacional (CARNEIRO JP e CABRAL H, 2016). Além disso, nesse período ocorrem alterações biológicas, vasculares e estruturais que acometem o Sistema Nervoso Central gerando modificações na liberação de neurotransmissores e hipotrofia cerebral (PAIXÃO YA, et al., 2019).

A depressão frequentemente ocorre associada a déficits cognitivos, apresentando alterações na memória, linguagem, atenção e na função executiva. A memória é a habilidade de armazenar, adquirir e registrar informações, sendo classificada em memória sensorial, onde informações são guardadas brevemente; memória de curto prazo, que permanecem na consciência por espaço menor; e memória de longo prazo, na qual o armazenamento de informações é permanente. A atenção é a capacidade de concentração numa determinada situação, enquanto a função executiva tem como finalidade a regulação e o controle de processamento de informações do cérebro, sendo responsável por iniciar uma atividade com finalidade determinada (FERREIRA JP, et al., 2019).

A depressão pode ser considerada fator de risco para o desenvolvimento de déficit cognitivo, estando ambos frequentemente relacionados. O diagnóstico diferencial é de extrema importância para o tratamento e o cuidado com a qualidade de vida do idoso (LAMPERT CDT e SCORTEGAGNA SA, 2017). Portanto, na suspeita de déficit cognitivo é importante a exclusão de causas secundárias, que pode ser estabelecido através da de uma história clínica detalhada, realização de exames laboratoriais, incluindo hemograma, ionagrama sérico, glicose em jejum, função renal, hepática, tireóidea, vitamina B12, folato e sorologia para sífilis. Para descartar a associação de deficiência cognitiva e o quadro depressivo, a escala de avaliação de depressão geriátrica é um instrumento decisivo (CARNEIRO JP e CABRAL YA, 2016).

\section{Insuficiência Renal}

A Insuficiência renal crônica (IRC) é um problema de saúde pública, caracterizada pela perda progressiva da capacidade de função dos néfrons e consequente perda da filtração sanguínea e homeostasia corporal (AGUIAR LK, et al., 2020). A IRC afeta cerca de $45 \%$ das pessoas com mais de 70 anos de idade. $O$ idoso do grupo de risco para esta doença possui diminuição da taxa de filtração glomerular em decorrência do avanço da idade (DELGADO MF, et al., 2017).

A modalidade de hemodiálise exige o comparecimento ao hospital ou clínica por cerca de duas a três vezes por semana, de duas a quatro horas, além disso, torna-se necessário uso de medicamentos e controle rígido de dieta, o que muitas vezes impossibilita a vida laboral do indivíduo e altera a sua percepção da vida como sujeito. Essas transformações acabam sendo deletérias para a formação da consciência, que é constituída de linguagem, pensamento e de emoções (CRUZ VFES, et al., 2016). Dessa forma, pacientes submetidos a hemodiálise possuem maior risco de comprometimento cognitivo (GUANARÉ VCSC, et al., 2016).

\section{CONSIDERAÇÕES FINAIS}

As deficiências cognitivas representam um grande problema de saúde pública, com consequências significativas para os idosos e seus familiares. $O$ estudo revela a existência de diversas etiologias primárias e secundárias. A DA está entre os tipos mais comuns, sendo o seu diagnóstico realizado através da exclusão de outras etiologias. Dessa forma, é fundamental o conhecimento dos diagnósticos diferenciais das deficiências cognitivas, visto que, o tratamento precoce pode reverter ou adiar a progressão para a demência.

\section{REFERÊNCIAS}

1. AGUIAR LK, et al. Fatores associados à doença renal crônica: inquérito epidemiológico da Pesquisa Nacional de Saúde. Rev. bras. epidemiol, 2020; 23: e200044. 
2. BEZERRA PK, et al. Déficit cognitivo: Proposição de cartilha para atenção ao idoso. Revista Brasileira de Pesquisa em Ciências da Saúde, 2016; 3(1): 1-10.

3. BRANDÃO BMLS, et al. Relação da cognição e qualidade de vida entre idosos comunitários: estudo transversal. Revista Brasileira de Enfermagem, 2020; 73(3): e20190030.

4. BRAUN NM, et al. Cianocobalamina como tratamento de doenças neuropsicomotoras em idosos com déficit de vitamina B12: revisão da literatura. Unisanta Health Science, 2017; 1(1): 80-87.

5. CAETANO LAO, et al. Alzheimer, sintomas e grupos: uma revisão integrativa. Vínculo, 2017; 14(2): 84-93.

6. CARDOSO SA, PAIVA I. Nutrição e Alimentação na prevenção e terapêutica da Demência. Acta Port Nutr, 2017; 11: 30-34.

7. CARNEIRO JP, CABRAL H. A linha ténue entre a demência e depressão no idoso: relato de caso. Rev Port Med Geral Fam, 2016; 32(2): 118-124.

8. CRUZ VFES, et al. A manutenção da vida laboral por doentes renais crônicos em tratamento de hemodiálise: uma análise dos significados do trabalho. Saúde soc, 2016; 25(4): 1050-1063.

9. DELGADO MF, et al. Fatores de risco e conhecimento de idosos sobre doença renal. Rev Rene (Online), 2017; 18(3): 361-367.

10. FALCO AD, et al. Doença de Alzheimer: Hipóteses Etiológicas e Perspectivas de Tratamento. Química Nova, 2016; 39(1): 63-80.

11. FAGUNDES TA, et al. Incapacidade funcional de idosos com demência. Cad. Ter. Ocup. UFSCar (Impr.), 2017; 25(1): 159-169.

12. FERREIRA JP, et al. Alterações de memória e funções executivas em pacientes com depressão. Psic., Saúde \& Doenças, 2019; 20(1): 114-121.

13. FRANÇA VF, et al. Indicadores de hábitos alimentares não saudáveis, concentração sérica das vitaminas $B 12$, ácido fólico e a cognição em idosos de Florianópolis. Tese (doutorado) - Centro de Ciências da Saúde. Universidade Federal de Santa Catarina, Florianópolis, 2016; 202p.

14. GUANARÉ, et al. Fatores associados à função cognitiva de pacientes com Doença Renal Crônica. Cad. Ter. Ocup. UFSCar (Impr.) 2016; 24(2): 287-296.

15. JORGE AJL, et al. Deficiência da Vitamina D e Doenças Cardiovasculares. Int. J. Cardiovasc. Sci., 2018; 31(4): 422432.

16. LAMPERT CDT, SCORTEGAGNA SA. Avaliação das condições de saúde e distorções cognitivas de idosos com depressão. Aval. psicol, 2017; 16(1): 48-58.

17. LANCHO MCR, BERCIANOS SG. Caracterização da linguagem nas variantes linguísticas da Afasia Progressiva Primária. Revista signos, 2020; 53(102):198-218.

18. MARTINS JT, et al. Efeitos da deficiência de vitamina B12 no cérebro. Inova Saúde, 2017; 6(1): $192-207$.

19. MENDONÇA FJP, et al.. Síndrome de Ekbom associada à demência por corpos de Lewy: relato de caso. Dement. neuropsicol., 2020; 14(1): 83-87.

20. MENEZES AV, et al. Efetividade de uma intervenção fisioterapêutica cognitivo-motora em idosos institucionalizados com comprometimento cognitivo leve e demência leve. Ciência \& Saúde Coletiva, 2016; 21: 3459-3467.

21. OLIVEIRA ACR, et al. O polimorfismo Bsml no gene do receptor da vitamina D está associado aos níveis de 25hidroxivitamina D em indivíduos com declínio cognitivo. Arq. Neuro-Psiquiatr., 2018; 76(11): 760-766.

22. PAIXÃO YA, et al. Declínio cognitivo e sintomas depressivos: Um estudo com idosos da universidade da maturidade. Revista humanidades e inovação, 2019; 6(11): 120-127

23. PANTOJA MAG, et al. Doença neurodegenerativa: demência frontotemporal. Sanid. Mil., 2020; 76(1): 39-40.

24. PRADO M, et al. Déficit cognitivo em idosos hospitalizados segundo Mini Exame do Estado Mental (MEEM): Revisão narrativa. Journal of Health Sciences, 2018; 20(2): 131-134.

25. SANTOS CS, et al. Fatores associados à demência em idosos. Ciência \& Saúde Coletiva, 2020; 25: 603-611.

26. SANTOS MD, BORGES SM. Percepção da funcionalidade nas fases leve e moderada da doença de Alzheimer: visão do paciente e seu cuidador. Rev. Brasileira de geriatria gerontol, 2015; 18(2): 339-349.

27. SHIBUYA $M$, et al. Neuroimagem na doença dos pequenos vasos cerebrais: atualização e novos conceitos. Dement. neuropsicol., 2017; 11(4):336-342. 